DOI: $10.3901 / J M E .2019 .10 .070$

\title{
大口径厚壁管热挤压模具磨损
}

\author{
王瑞麒 李永堂 \\ （太原科技大学金属材料成形理论与技术山西省重点实验室 太原 030024)
}

\begin{abstract}
摘要: 为探讨大口径厚壁管热挤压模具的磨损规律, 以 ASME SA-335P91 钢、AISI H13 钢为研究对象, 结合实际试验与有 限元模拟, 分析温度与保温时间对模具磨损的影响情况。从模具工作条件观察得到: 在金属坏料与模具接触初期, 模具表面 温升对磨损影响较大; 在金属坏料与模具处于热平衡状态时, 该状态持续时间与当前温度是影响磨损的主要因素。在金属坏 料与模具接触初期得到了温度-硬度方程、温度-磨损系数方程; 在热平衡状态下得到了时间-硬度方程、温度-磨损系数方程。 结合 Archard 磨损理论, 得到了适用于大口径厚壁管热挤压模具的磨损规律。最后对有限元软件进行二次开发, 结果表明, 预测值与高温磨损试验值基本一致，可为该类模具磨损优化设计提供理论支持。
\end{abstract}

关键词: 大口径厚壁管；热挤压模具；磨损规律；高温硬度试验；高温磨损试验；模具软化试验

中图分类号: TG375

\section{Wear of Hot Extrusion Die for Large Diameter Thick-walled Pipes}

\section{WANG Ruiqi LI Yongtang}

(Shanxi Key Laboratory of Metallic Material Forming Theory and Technology,

Taiyuan University of Science and Technology, Taiyuan 030024)

\begin{abstract}
In order to investigate the wear regularity of hot-extrusion die for large-diameter thick-walled pipe, ASME SA-335P91 steel and AISI H13 steel are taken as research objects. Combined with actual tests and finite element simulation, the influence of temperature and holding time on die wear is analyzed. Obtained from the working conditions of the mold: in the initial contact of the metal billet with the die, the temperature rise of the die surface has a great influence on the wear; when the metal billet and the die are in thermal equilibrium, the duration of the state and the current temperature are the main factors affecting the wear. The temperature-hardness equation and the temperature-wear coefficient equations are obtained at the initial contact of the metal billet with the die. The time-hardness equations and the temperature-wear coefficient equation are obtained under thermal equilibrium. Combined with the Archard wear theory, the wear regularity of hot extrusion die for large diameter thick wall pipe is obtained. Finally, the secondary development of the finite element software shows that the predicted value is basically consistent with the high temperature wear test value, which can provide theoretical support for the optimization design of the die wear.
\end{abstract}

Key words: large diameter thick-walled pipe; hot extrusion die; wear regularity; high temperature hardness test; high temperature wear test; die-softening test

\section{0 前言}

大口径厚壁管(尺寸范围外径 $\phi 406 \sim 1320$ mm、 壁厚 20〜240 mm、管长 6 12 m) 是核电、石油等 行业的核心基础部件，主流生产工艺是热挤压成 形 ${ }^{[1-2]}$ 。相对于普通管件, 大口径厚壁管的成形时间较 长, 根据管件尺寸一般在 3 $8 \mathrm{~min}$ 之间。并且在成形 时间内, 模具需要在高温下承受坏料塑性变形、流动 产生的挤压力与摩擦力。从某生产企业报废模具观测

20180522 收到初稿, 20190121 收到修改稿
可知, 大口径厚壁管热挤压模具的磨损非常严重。 摩擦磨损现象广泛存在于生产制造中, 因有时 造成巨大的经济损失而受到人们极大的关注 ${ }^{[3]}$ 。 1953 年, $\mathrm{ARCHARD}^{[4]}$ 基于粘着磨损理论推导出简 单的磨损量计算公式, 该公式中含有磨损系数 $k_{s}$, 如式(1)所示

$$
W=k_{s} \frac{L P}{H}
$$

式中, $W$ 代表磨损量; $k_{s}$ 为磨损系数; $P$ 为法向载 荷; $L$ 为摩擦行程; $H$ 为模具表面硬度。

在此基础上, 众多学者对 Archard 磨损模型进 行修正。KANG 等 ${ }^{[5-6]}$ 通过建立模具表面硬度与模具 
总工作时间、温度的函数方程, 基于 Archard 磨损 模型提出了一种考虑热软化的磨损模型; LEE 等 ${ }^{[7]}$ 假设 Archard 磨损模型仍然适合于温锻成形, 但是 模具表面硬度和磨损系数随温度变化, 并结合磨损 试验, 得出高温下 H13 钢的磨损系数、模具表面硬 度与温度的关系式; BEHRENS ${ }^{[8]}$ 通过测量大量工业 锻造模具的磨损, 建立回火参数与硬度的方程, 对 Archard 磨损模型进行改进, 随后将该模型导入有限 元软件进行了磨损预测, 预测磨损深度与实际测量 基本一致; $\mathrm{ABACHI}$ 等 ${ }^{[9]}$ 通过有限元模拟软件 MSC.SuperForge 对闭式热锻模进行磨损研究。并对 比工厂实际测量结果, 对 Archard 磨损模型的磨损 系数进行了修正, 得到了计算闭式模锻模具磨损的 修正 Archard 磨损模型。总体来看, 模具硬度与磨 损系数是影响磨损的主要因素, 而温度则是影响硬 度与磨损系数的直接因素。

在大口径厚壁管实际生产过程中, 坏料加热温 度通常远高于模具的预热温度, 且成形时间较长。 通过有限元模拟计算与实际生产中的观测, 发现模 具表面在与金属坏料接触后, 温度迅速上升, 上升 速率随温度升高而逐渐降低, 最后均稳定在一定温 度区间内。此时, 模具表面与金属坏料达到近似热 平衡状态, 并一直保持到挤压过程结束。

从而可知, 在研究温度对大口径厚壁管模具磨 损影响时, 应分为两种情况分别进行讨论: 在金属 坏料与模具接触初期, 温度迅速升高, 模具磨损主 要受温度变化的影响; 在模具与坏料处于近似热平 衡状态时, 温度变化不大, 模具磨损主要受该状态 持续时间的影响。

基于以上分析, 以 Archard 磨损模型为基础, 以热挤压模具常用材料 AISI H13 模具钢、大口径厚 壁管典型耐热材料 ASME SA-335P91 合金钢为研究 对象, 考虑温度在热挤压各阶段影响磨损的主要形 式, 设计了高温硬度试验、高温磨损试验、模具软 化试验。其中, 模具软化试验是模具材料在各温度 段保温不同时间后空冷到室温时对其硬度进行测量 获得其硬度随保温时间的变化趋势并假设这种趋势 可以表征高温下硬度随保温时间的变化趋势。综合 分析三种试验结果, 得到了适用于大口径厚壁管热 挤压模具的磨损规律, 并借助有限元模拟软件预测 了模具磨损情况。

\section{1 高温试验}

\section{1 试验材料}

本次试验所用 AISI H13 钢原始组织为退火状
态, ASME SA-335P91 钢为铸态。其中, P91 钢直 接加工成直径为 $4 \mathrm{~mm}$ 的钢珠, 作为高温磨损试验 的对磨材料。同时, 对 H13 钢进行常规热处理 ${ }^{[10-11]}$, 使其内部组织与实际生产中热挤压模具相似。热处 理工艺流程如图 1 所示。将热处理好的 H13 钢线切 割加工成 7 个 $\phi 10 \mathrm{~mm} \times 12 \mathrm{~mm}$ 圆柱形试样, 7 个 $\phi 40 \mathrm{~mm} \times 10 \mathrm{~mm}$ 圆盘试样和 35 个 $\phi 4 \mathrm{~mm} \times 2 \mathrm{~mm}$ 圆 盘试样。三种试样分别用于高温硬度试验、高温磨 损试验和模具软化试验。表 1 是 AISI H13 钢和 ASME SA-335P91 两种钢的化学成分。

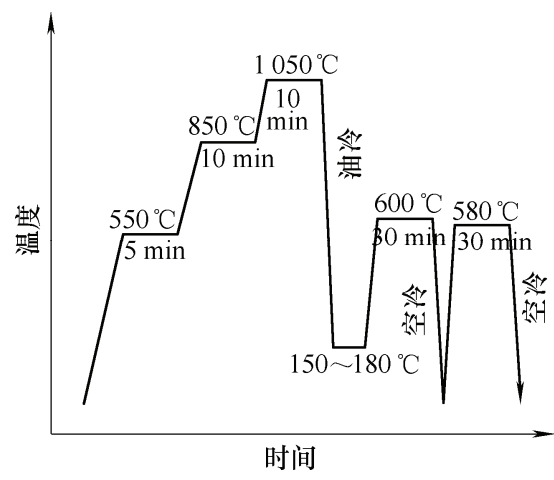

图 1 AISI H13 钢热处理工艺图

表 1 AISI H13 与 ASME SA-335P91 的化学成分(质量分数) \%

\begin{tabular}{ccccc}
\hline 材料 & $\mathrm{C}$ & $\mathrm{Mn}$ & $\mathrm{Si}$ & $\mathrm{Cr}$ \\
\hline AISI H13 & $0.32 \sim 0.45$ & $0.20 \sim 0.50$ & $0.80 \sim 1.20$ & $4.75 \sim 5.50$ \\
ASME SA-335P91 & $0.08 \sim 0.12$ & $0.30 \sim 0.60$ & $0.20 \sim 0.50$ & $8.00 \sim 9.50$ \\
\hline \hline 材料 & Mo & $\mathrm{V}$ & $\mathrm{P}$ & $\mathrm{S}$ \\
\hline AISI H13 & $1.10 \sim 1.75$ & $0.80 \sim 1.20$ & $\leqslant 0.030$ & $\leqslant 0.03$ \\
ASME SA-335P91 & $0.85 \sim 1.05$ & $0.18 \sim 0.25$ & $\leqslant 0.020$ & $\leqslant 0.020$ \\
\hline
\end{tabular}

\section{2 高温硬度试验}

许多学者对不同温度下 $\mathrm{H} 13$ 钢的硬度变化趋势 进行了大量的研究 ${ }^{[12-14]}$ 。从这些研究结果来看, H13 钢在 $500 \sim 600{ }^{\circ} \mathrm{C}$ 之间具有二次硬化效应。当温度超 过 $600{ }^{\circ} \mathrm{C}$ 时, 硬度开始急剧下降。

在制造大口径厚壁管时, 热挤压模具通常被预 热至 $300{ }^{\circ} \mathrm{C}$, 坏料通常加热至 $1100{ }^{\circ} \mathrm{C}$ 以上。当坏 料与模具接触时, 模具表面温度在很短的时间内被 加热到高温, 局部温度将超过 $600{ }^{\circ} \mathrm{C}$ 。从有限元模 拟结果来看, 模具的局部温度可达到 $800{ }^{\circ} \mathrm{C}$ 左右。 因此, 试验温度选择分别为 $300{ }^{\circ} \mathrm{C}, 450{ }^{\circ} \mathrm{C}, 500{ }^{\circ} \mathrm{C}$, $550{ }^{\circ} \mathrm{C}, 600{ }^{\circ} \mathrm{C}, 700{ }^{\circ} \mathrm{C}$ 和 $800{ }^{\circ} \mathrm{C}$ 。使用 ARCHIMEDES HTV-PHS30 高温维氏硬度计测量不 同温度下样品的表面硬度。试验载荷为 $29.4 \mathrm{~N}$ 。试验 结果用于分析 H13 钢表面硬度与温度之间的关系。

\section{3 高温磨损试验}

在 Archard 磨损模型中, 磨损系数被定义为产 生磨粒的概率 ${ }^{[15]}$ 。H13 钢与不同材料对磨产生磨粒 
的概率是不同的。为了获得 H13 钢与 P91 钢对磨情 况下的磨损系数, 设计了球盘式高温磨损试验。并 使用 HT-1000 高温摩擦磨损试验机测试 H13 钢的表 面耐磨性能: 首先, 将 H13 钢磨盘固定在炉内, 待 炉内温度保持在指定温度下时装载加载杆, 磨盘表 面与加载杆内 P91 钢珠发生对磨而产生磨损。试验 设备如图 2 所示, 试验条件如表 2 所示。试验完成 后, 使用超声波清洗机对磨盘进行清洗。然后, 使 用 MT-500 探针材料表面磨损测试仪(扫描探针纵向 分辨率: $0.02 \mu \mathrm{m}$ ) 测试磨损宽度和磨损深度, 如图 3

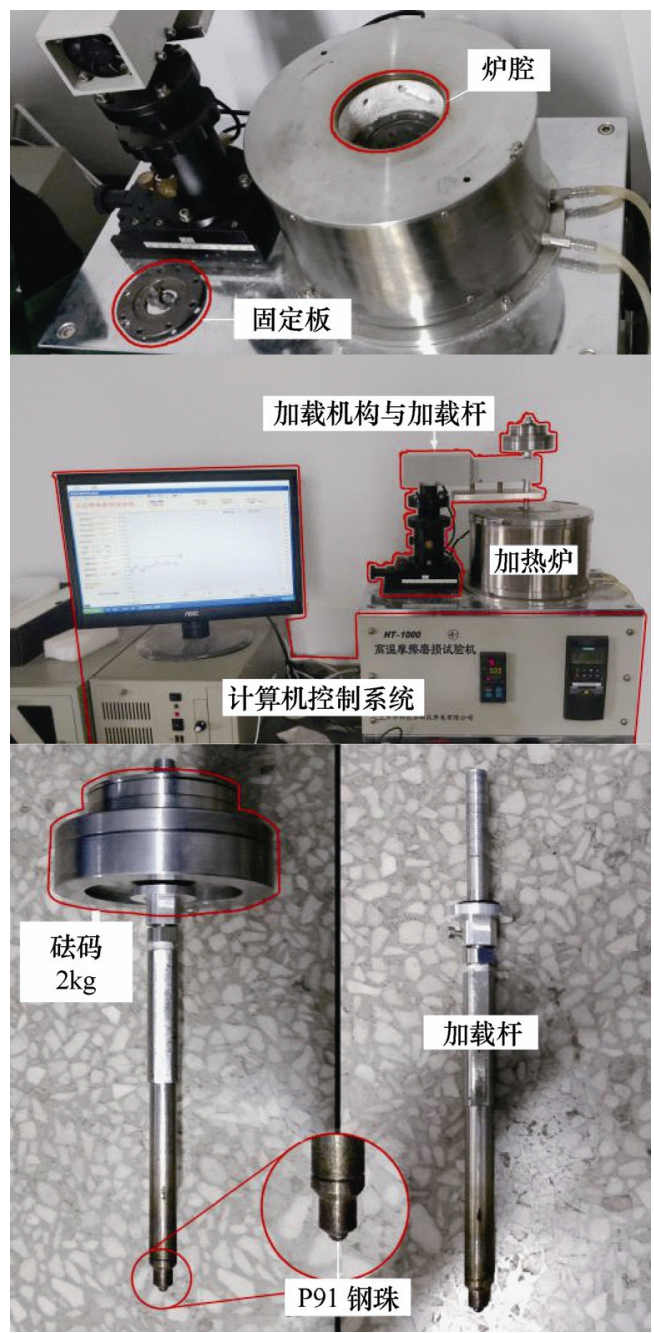

图 2 HT-1000 高温摩擦磨损试验机

表 2 高温磨损试验条件

\begin{tabular}{lc}
\hline \multicolumn{1}{c}{ 名称 } & 参数或条件 \\
\hline 对磨材料 & ASME SA-335P91 \\
磨盘材料 & AISI H13 \\
载荷 $/ \mathrm{N}$ & 20 \\
线速度 $/(\mathrm{mm} / \mathrm{s})$ & 500 \\
旋转半径 $/ \mathrm{mm}$ & 8 \\
滑动距离 $/ \mathrm{m}$ & 300 \\
温度 $/{ }^{\circ} \mathrm{C}$ & $300,450,500,550,600,700 、 800$ \\
润滑 & 无 \\
气氛 & 大气 \\
\hline
\end{tabular}

所示。测试结果用于分析 H13 钢在不同预热温度下 的磨损系数。

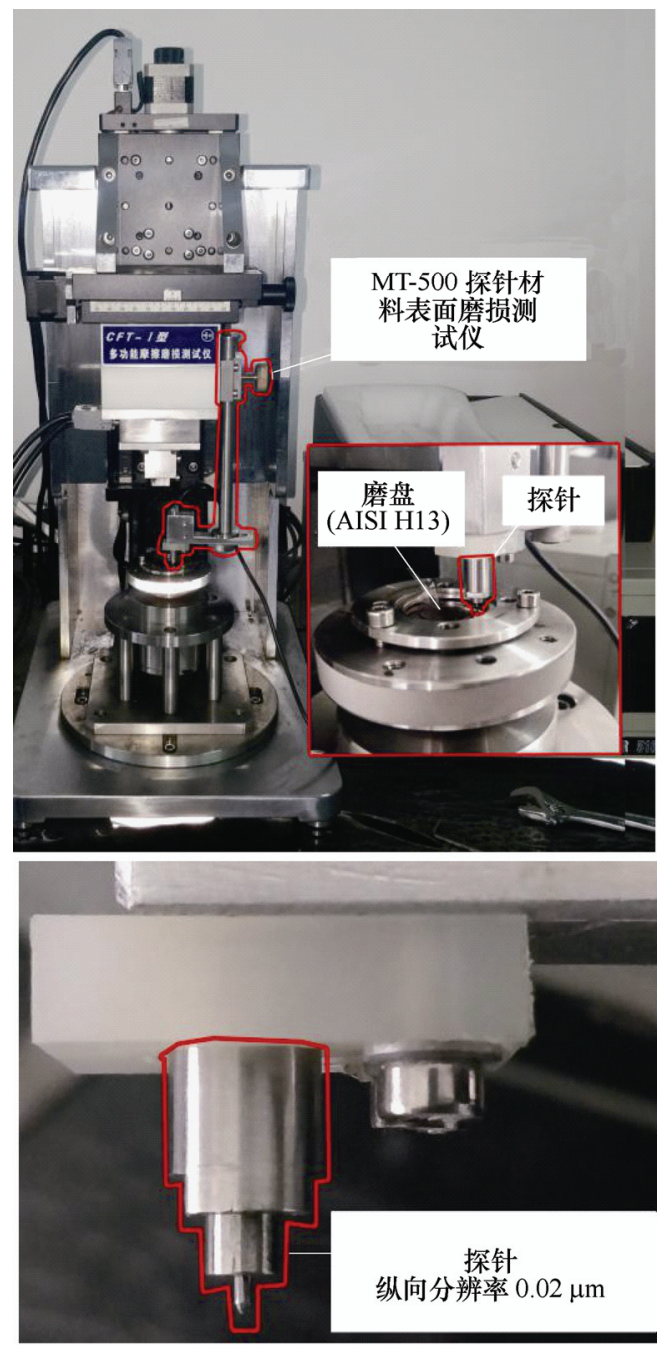

图 3 MT-500 探针材料表面磨损测试仪

\section{4 模具软化试验}

为了研究模具表面在热平衡状态下的硬度变 化, 依照高温磨损试验中选择的温度值将 H13 钢试 样分成七组, 每组 5 个样本。根据前文对大口径厚 壁管成形时间的分析, 一组的每个样本分别在指定 的温度下保温 $2 \mathrm{~min}, 4 \mathrm{~min}, 6 \mathrm{~min}, 8 \mathrm{~min}, 10 \mathrm{~min}$, 然 后依次取出样品进行空冷。热处理过程如图 4 所示。 随后, 用 HR-150A 型洛氏硬度计测量试样的表面硬 度。当保温温度超过 $600{ }^{\circ} \mathrm{C}$ 时, 由于表面软化严重, 用 HBS-62.5 布氏硬度计测量试样表面硬度(该款硬 度计可直接显示维氏硬度)。为了便于计算, 所测得 的洛氏硬度值使用等式(2)转换成维氏硬度, 该等式 根据 ISO 18265:2013(E) ${ }^{[16]}$ 中内容使用 Matlab 软件 拟合得出。试验结果用于分析模具在热平衡状态下 的表面硬度随保温时间的变化趋势。

$$
\begin{gathered}
H V=-2.023 \times 10^{-4} \times H R C^{3}+0.02091 \times H R C^{2}+ \\
9.348 \times H R C-0.7253
\end{gathered}
$$




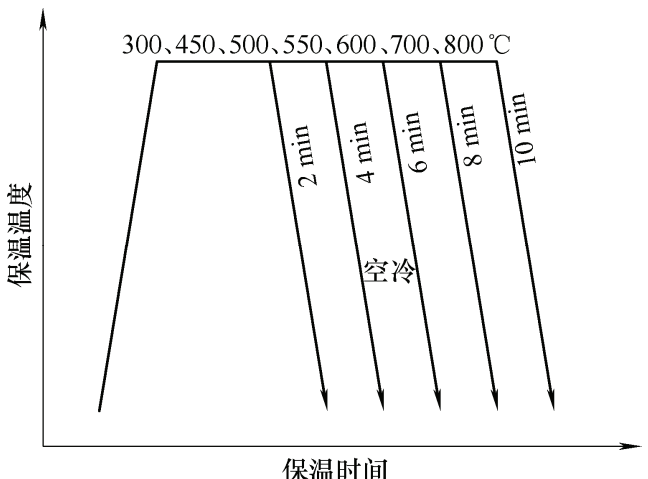

图 4 模具软化试验热处理工艺图

\section{2 结果与讨论}

\section{1 试验结果分析}

高温硬度试验中, 每个试样均测量五次, 结果 取平均值。总体来看, $\mathrm{H} 13$ 钢的硬度随温度的升高 而降低。在 $600{ }^{\circ} \mathrm{C}$ 时, 试样出现了二次硬化效应。 如图 5 所示。

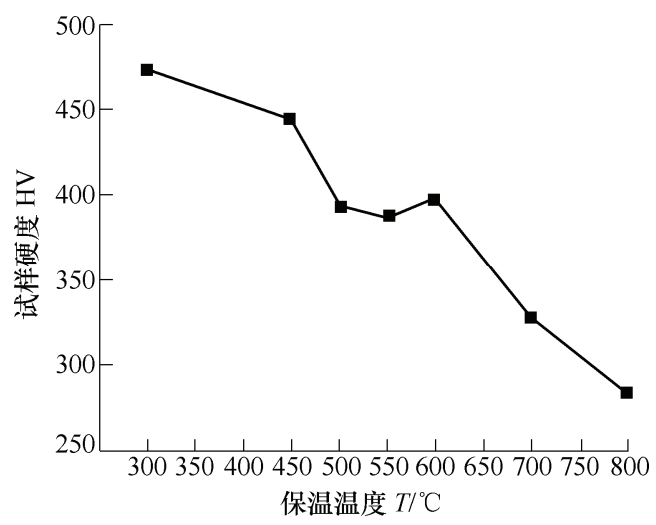

图 5 AISI H13 高温硬度曲线

如图 6 所示, 在保温温度为 $300 \sim 550{ }^{\circ} \mathrm{C}$ 范围内 时, 保温时间对 $\mathrm{H} 13$ 钢的硬度影响不大。但是, 当 温度维持在 $600{ }^{\circ} \mathrm{C}$ 时, 保温时间已经开始影响 $\mathrm{H} 13$ 钢的硬度。当保温温度升高到 $700{ }^{\circ} \mathrm{C}$ 和 $800{ }^{\circ} \mathrm{C}$ 时, 随着保温时间增加, H13 钢的硬度显著下降(图 6 中 的 RT 表示 H13 钢在室温下测得的硬度)。

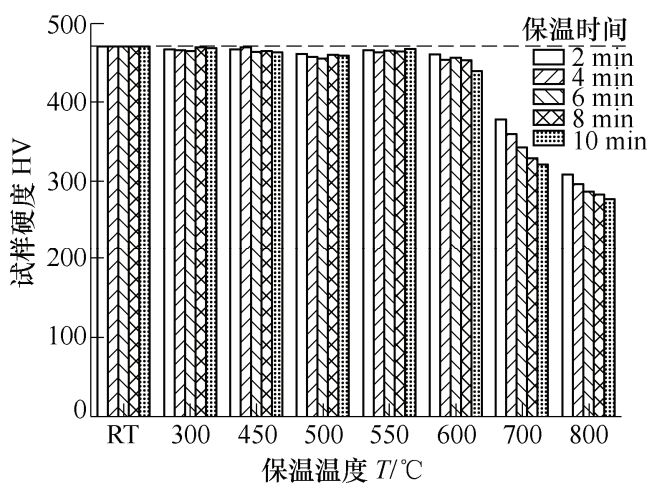

图 6 模具软化试验结果
测量磨盘的磨损深度时, 磨盘被分成八个区域, 每个区域测量三次，最后计算出所有测量值的平均 值用于表示该磨盘的磨损深度，如图 7、8 所示。

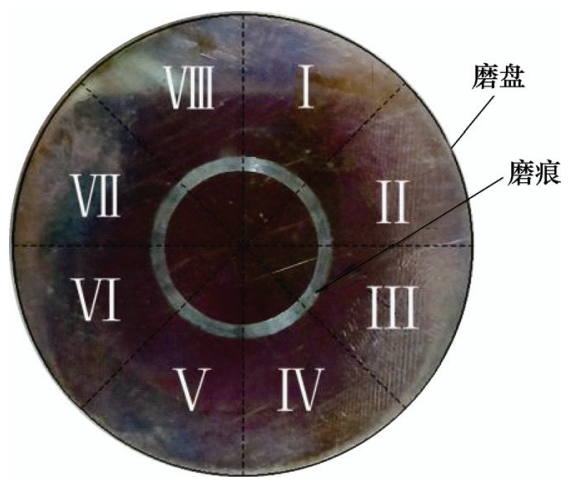

图 7 磨盘分区图

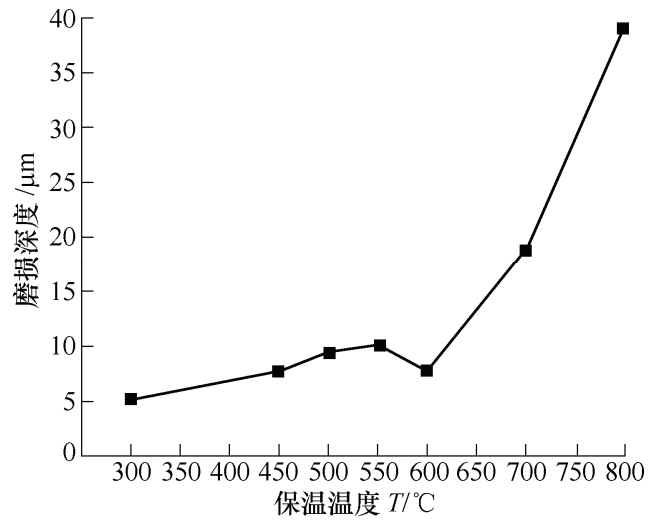

图 8 高温磨损试验结果图

从图 8 可以看出, 磨损深度随着温度的升高而 增加。但在 $600{ }^{\circ} \mathrm{C}$ 时, 磨损深度减小。这是因为 $\mathrm{H} 13$ 钢在该温度范围内出现了二次硬化现象, 硬度增加, 耐磨性提高。当温度超过 $600{ }^{\circ} \mathrm{C}$ 时, 由于硬度急剧 下降，磨损深度迅速增加。从图 9a 可看出, $\mathrm{H} 13$ 钢 在 $300{ }^{\circ} \mathrm{C}$ 时, 磨痕相对较浅且清晰; 对比 $600{ }^{\circ} \mathrm{C}$, 尽管磨损深度从数值上看与 $300{ }^{\circ} \mathrm{C}$ 差别不大, 但磨 痕的表面形貌却发生了很大改变; 从图 9c、9d 可看 出, 温度超过 $600{ }^{\circ} \mathrm{C}$ 时, 试样的磨损更加剧烈, 磨 痕深度明显加深, 呈现出犁沟状。

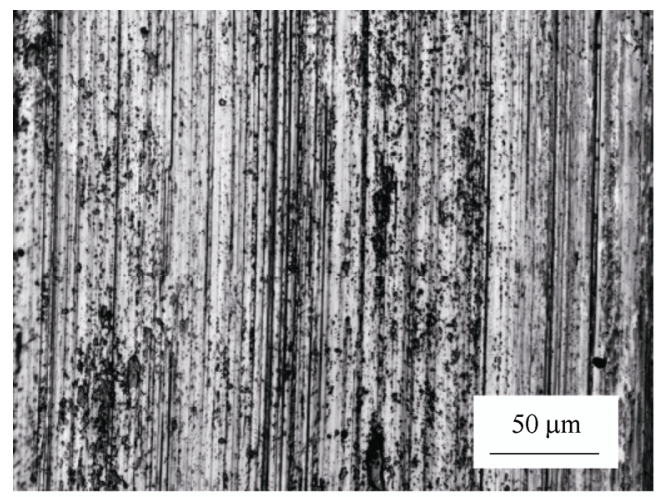

(a) $300^{\circ} \mathrm{C}$ 


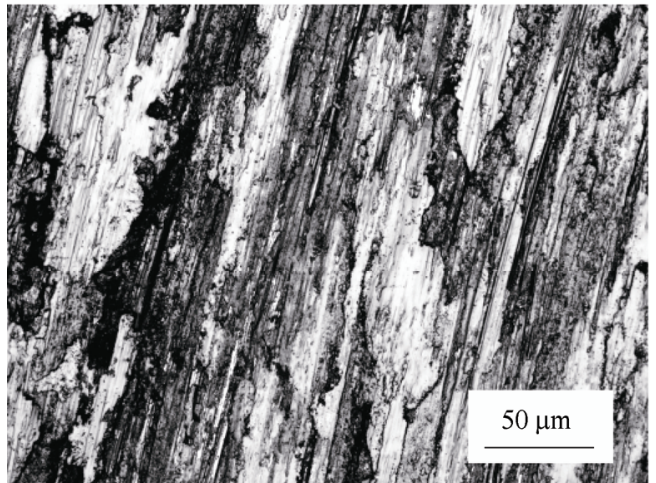

(b) $600{ }^{\circ} \mathrm{C}$

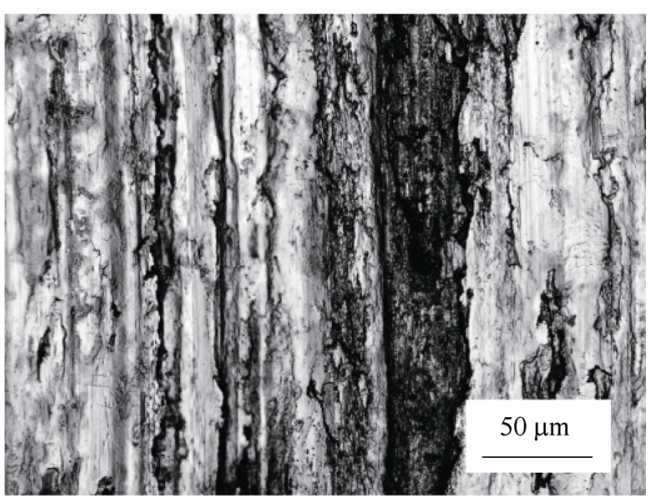

(c) $700^{\circ} \mathrm{C}$

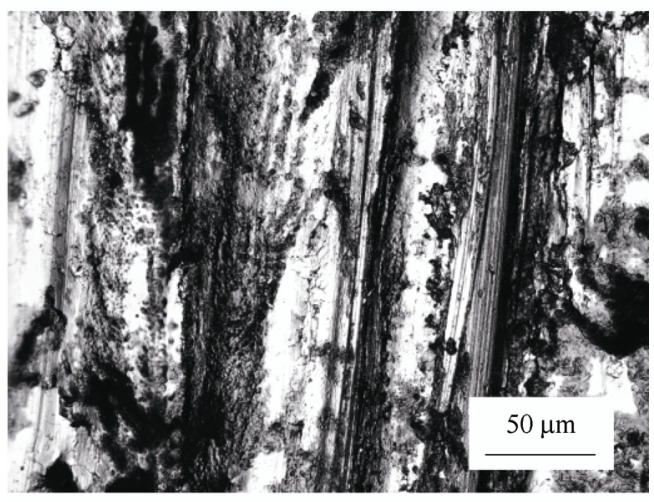

(d) $800^{\circ} \mathrm{C}$

图 9 H13 钢在不同温度下的磨损表面形貌

\section{2 磨损规律建立}

综合以上三种试验结果, 可以发现 H13 钢 的性能在 $600{ }^{\circ} \mathrm{C}$ 左右发生了很大变化: $600{ }^{\circ} \mathrm{C}$ 之 前试样表面硬度主要受温度变化影响, 而对保温 时间并不敏感, 磨损深度随着温度升高缓慢上 升。当温度升高到 $600{ }^{\circ} \mathrm{C}$ 之后, 试样表面硬度不 仅受到温度变化的影响, 而且保温时间越长硬度 越低, 磨损深度急剧升高。因此, 在 $300 \sim 600{ }^{\circ} \mathrm{C}$ 温度段建立温度-硬度方程、温度一磨损系数方 程, 如图 10 所示; 在 $600 \sim 800{ }^{\circ} \mathrm{C}$ 温度段时, 建 立温度-磨损系数方程, 并基于高温硬度试验结 果与模具软化试验得到的 $700{ }^{\circ} \mathrm{C}$ 与 $800{ }^{\circ} \mathrm{C}$ 下试
样硬度随时间的变化规律, 建立时间-硬度方程 如图 11 所示。
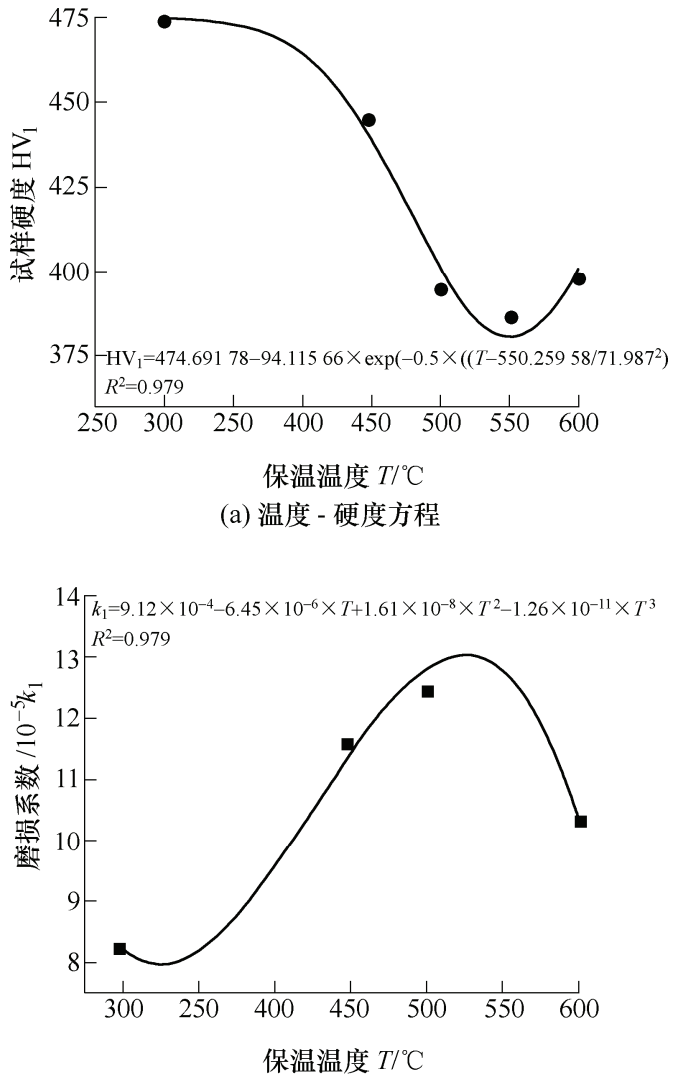

(b) 温度 - 磨损系数方程

图 $10300{ }^{\circ} \mathrm{C} \sim 600{ }^{\circ} \mathrm{C}$ 试验结果分析

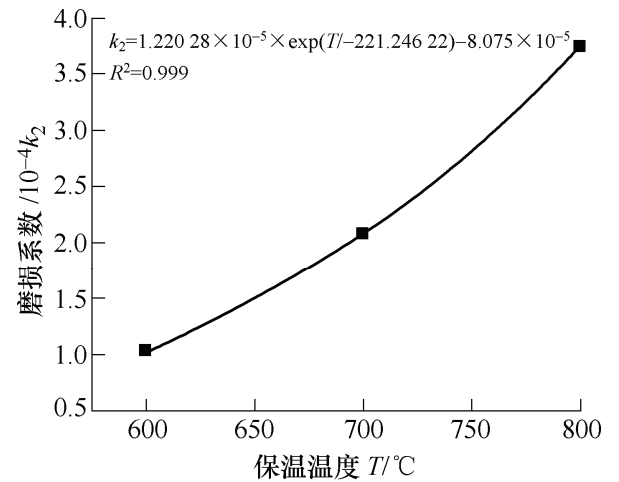

(a) 温度 - 磨损系数方程

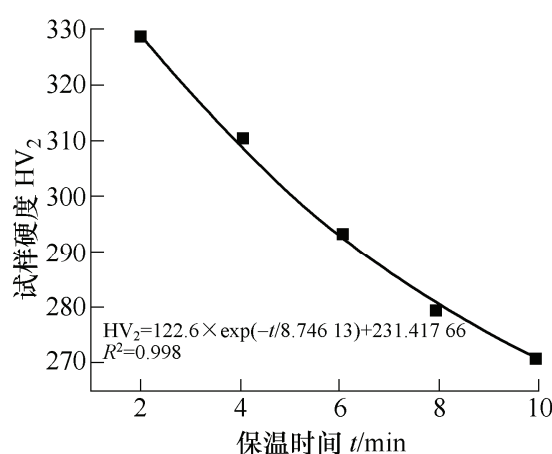

(b) 时间 - 硬度方程 $\left(600 \sim 700^{\circ} \mathrm{C}\right)$ 


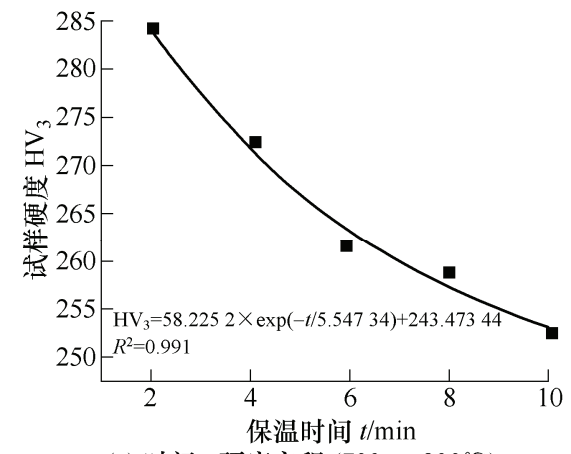

(c) 时间 - 硬度方程 $\left(700 \sim 800^{\circ} \mathrm{C}\right)$

图 $11600{ }^{\circ} \mathrm{C} \sim 800{ }^{\circ} \mathrm{C}$ 试验结果分析

所有方程如下。

温度范围: $300 \sim 600{ }^{\circ} \mathrm{C}$ 。

温度-硬度方程

$$
\begin{gathered}
H V_{1}=474.69178-94.11566 \times \\
\exp \left(-0.5 \times[(T-550.25958) / 71.987]^{2}\right)
\end{gathered}
$$

温度-磨损系数方程

$$
\begin{gathered}
k_{1}=4.66177 \times 10^{-6}+7.47224 \times 10^{-6} \times \\
\exp \left(-0.5 \times[(T-531.9332) / 80.133]^{2}\right)
\end{gathered}
$$

式中, $T$ 为模具表面温度。

温度范围: $600 \sim 700{ }^{\circ} \mathrm{C}$ 。

时间-硬度方程

$$
H V_{2}=122.6 \times \exp (-t / 8.74613)+231.41766
$$

式中, $t$ 为保温时间。

温度范围: $700 \sim 800{ }^{\circ} \mathrm{C}$ 。

时间-硬度方程

$$
H V_{3}=58.2252 \times \exp (-t / 5.54734)+243.47344 \text { (6) }
$$

式中, $t$ 为保温时间。

温度范围: $600 \sim 800{ }^{\circ} \mathrm{C}$ 。

温度-磨损系数方程

$$
\begin{gathered}
k_{2}=1.22028 \times 10^{-5} \times \exp (-T / 221.24622)- \\
8.075 \times 10^{-5}
\end{gathered}
$$

式中, $T$ 为模具表面温度。

\section{3 有限元模拟}

有限元分析的主要目的是验证模具软化试 验假设的正确性与磨损公式的实用性, 便于在工 程中使用该公式。因此, 基于 ABSOFT 8.0 编译 器, 使用 Fortran77 语言将得到的方程式写入 Deform3D V10.0 FEM。有限元模拟条件与高温磨 损试验条件一致。并且, 有限元结果的处理方式 也与高温磨损试验结果处理方式一致。模拟结果 如图 12 所示。

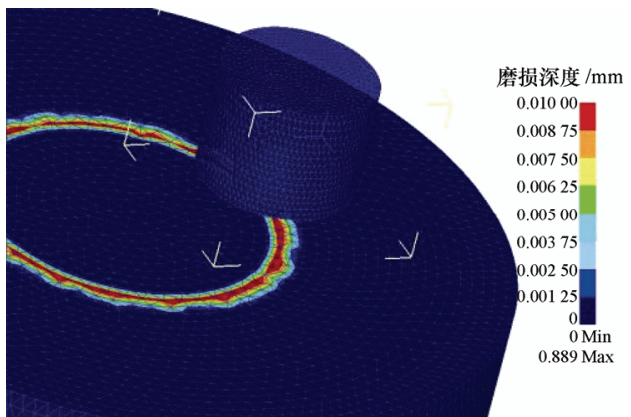

图 12 磨损量有限元模拟结果

从图 13 中 $700{ }^{\circ} \mathrm{C}$ 和 $800{ }^{\circ} \mathrm{C}$ 的模拟结果来看, 它 与实际的试验结果基本一致。这可以解释模具软化 测试假设的正确性。因为, 在实际的高温磨损试验 中, 磨盘一直处于高温状态。随着保温时间的增加, 磨盘的硬度降低。而在磨损时间, 载荷等条件确定 的前提下, 磨盘硬度的变化是影响最后磨损深度的 主要因素。同时, Deform 软件通过新磨损公式计算 出的磨损深度与实际测量值差别很小且趋势相同。 这代表在有限元模拟中，磨盘硬度的变化趋势与实 际高温磨损试验磨盘硬度的变化趋势一致。证明了 模具软化试验测得硬度变化趋势可以表征高温下硬 度随保温时间的变化趋势假设的正确性。

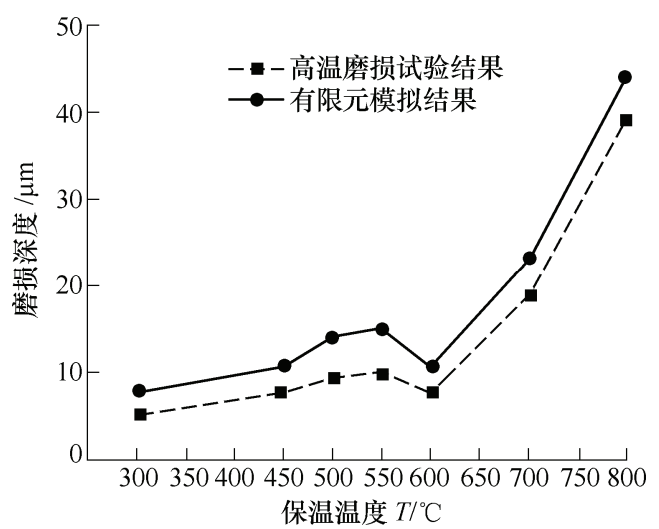

图 13 磨损深度模拟结果与实际试验结果对比

总体来看，有限元模拟结果整体变化趋势与实 际试验一致, 磨损深度预测值略微偏大, 这有利于 提高后期模具优化效果，提高模具使用寿命。证明 了新磨损规律的实用性。

\section{3 结论}

(1) 温度对大口径厚壁管挤压成形模具磨损影 响分为两个阶段: 在金属坏料与模具接触初期模具 受热低温阶段, 模具磨损主要受温度变化的影响; 在模具与坏料处于热平衡状态时的高温阶段，模具 磨损主要受热平衡状态持续时间影响。

(2) 通过高温硬度试验、高温磨损试验、模具 
软化试验, 分别得到了 AISI H13 钢在高温条件下不 同接触受热时间的硬度变化趋势与耐磨量变化规 律, 通过数据处理, 得到了成形 P91 材料的 H13 钢 基于 Archard 模型的磨损系数与硬度分别随模具表 面温度与接触时间的变化关系。

(3) 通过对 Deform 软件进行二次开发, 将本文 研究获得的磨损系数与硬度分别随模具表面温度与 接触时间的变化关系嵌入到软件中, 采用与试验相 同的温度与接触压力等条件, 基于 Archard 模型, 模拟了试验过程, H13 磨盘磨损量有限元模拟结果 与实际试验结果基本一致。同时也验证了模具软化 试验假设的正确性。

\section{参 考 文 献}

[1] 惠恬静, 雷步芳, 李永堂, 等. P91 厚壁管铸挤复合成 形工艺参数对组织和力学性能的影响 [J]. 机械工程学 报，2018，54(12): 156-164

HUI Tianjing, LEI Bufang, LI Yongtang, et al. Effects of processing parameters of casting-extruding on microstructure and mechanical properties of P91 thick-wall steel tube[J]. Journal of Mechanical Engineering, 2018, 54(12): 156-164.

[2] 贾璐, 李永堂, 李振晓. 耐热合金钢 P91 热变形过程静 态及亚动态再结晶行为 [J]. 机械工程学报, 2017, 53(8): 58-67.

JIA Lu, LI Yongtang, LI Zhenxiao. Static and metadynamic recrystallization behaviors of heat-resistant P91 alloy during hot deformation[J]. Journal of Mechanical Engineering，2017，53(8): 58-67

[3] 温诗铸. 材料磨损研究的进展与思考 [J]. 摩擦学学报, 2008, 28(1): 1-5.

WEN Shizhu. Progress and thinking on material wear research[J]. Tribology, 2008, 28(1): 1-5.

[4] ARCHARD J F. Contact and rubbing of flat surfaces[J]. Journal of Applied Physics, 1953, 24(8): 981-988.

[5] KANG J H, PARK I W, JAE J S, et al. A study on a die wear model considering thermal softening(I) : Construction of the wear model[J]. Journal of Materials Processing Technology, 1999, 96(1-3): 53-58.

[6] KANG J H, PARK I W, JAE J S, et al. A study on die wear model considering thermal softening (II) : Application of the suggested wear model[J]. Journal of
Materials Processing Technology, 1998, 94(2-3): 183-188.

[7] LEE R S, JOU J L. Application of numerical simulation for wear analysis of warm forging $\operatorname{die}[\mathrm{J}]$. Journal of Materials Processing Technology, 2003, 140(1-3): 43-48.

[8] BEHRENS B A. Finite element analysis of die wear in hot forging processes[J]. CIRP Annals-Manufacturing Technology, 2008, 57(1): 305-308.

[9] ABACHI S, AKKÖK M, GÖKLER M İ. Wear analysis of hot forging dies[J]. Tribology International, 2010, 43(1-2): 467-473.

[10] YAN G, HUANG X, WANG Y, et al. Effects of heat treatment on mechanical properties of h13 steel[J]. Journal of Hefei University of Technology，2011， 52(7-8): 393-395.

[11] BAHRAMI A, ANIJDAN S H M, GOLOZAR M A, et al. Effects of conventional heat treatment on wear resistance of AISI H13 tool steel[J]. Wear, 2005, 258(5): 846-851.

[12] NELSON E, KOHLI A, POIRIER D R. Hardness of H13 tool steel after non-isothermal tempering[J]. Journal of Materials Engineering \& Performance, 2018: 1-6.

[13] 周银, 花华平, 张秋阳, 等. H13 钢对磨不同回火工艺 的 $\mathrm{Cr} 12 \mathrm{MoV}$ 钢的磨损行为 [J]. 材料热处理学报, 2015, 36(s1): 208-212.

ZHOU Yin, HUA Huaping, ZHANG Qiuyang, et al. Wear behavior of $\mathrm{H} 13$ steel sliding against $\mathrm{Cr} 12 \mathrm{MoV}$ steel with different tempering process $[\mathrm{J}]$. Ansactions of Materials and Heat Treatment, 2015，36(s1): 208-212.

[14] NELSON E, KOHLI A, POIRIER D R. Hardness of H13 tool steel after non-isothermal tempering[J]. Journal of Materials Engineering \& Performance, 2018: 1-6.

[15] 桂长林. Archard 的磨损设计计算模型及其应用方法 [J]. 润滑与密封, 1990(1): 12-21

GUI Changlin. Archard's wear design calculation model and its application method[J]. Lubrication Engineering, 1990(1): 12-21.

[16] International organization for standardization. ISO 18265: 2013(E) Metallic materials Conversion of hardness values[S]. Switzerland: SGS SA, 2013.

作者简介: 王瑞麒, 男, 1994 年出生。主要研究方向为材料先进制造 技术。

E-mail: rickywang6677@163.com

李永堂(通信作者), 男, 1957 年出生, 博士, 教授, 博士研究生导师。 主要研究方向为材料先进制造技术。

E-mail: liyongtang@tyust.edu.com 